\title{
Derivative Expansion of One-Loop Effective Energy of Stiff Membranes with Tension
}

\author{
M. E. S. Borelli*, H. Kleinert ${ }^{\dagger}$, and Adriaan M. J. Schakel ${ }^{\ddagger}$ \\ Institut für Theoretische Physik \\ Freie Universität Berlin \\ Arnimallee 14, 14195 Berlin.
}

(October 7, 2018)

\begin{abstract}
With help of a derivative expansion, the one-loop corrections to the energy functional of a nearly flat, stiff membrane with tension due to thermal fluctuations are calculated in the Monge parametrization. Contrary to previous studies, an arbitrary tilt of the surface is allowed to exhibit the nontrivial relations between the different, highly nonlinear terms accompanying the ultraviolet divergences. These terms are shown to have precisely the same form as those in the original energy functional, as necessary for renormalizability. Also infrared divergences arise. These, however, are shown to cancel in a nontrivial way.
\end{abstract}

PACS: 82.65.Dp, 68.35.Rh

Keywords: Fluid membranes; Derivative expansion; Renormalization

Due to their small surface tension, fluid membranes (see Ref. [1] for reviews) are subject to strong thermal undulations. The energy of such a membrane is usually modeled by the local expression [2, 3 ]

$$
E_{0}=\mu_{0} \int \mathrm{d} S+\frac{1}{2} \int \mathrm{d} S\left(\kappa_{0} H^{2}+\bar{\kappa}_{0} K\right)
$$

where $\mathrm{d} S$ are the surface elements, while $H$ and $K$ denote (twice) the mean and the Gaussian curvature of the membrane surface, respectively. In terms of the principal radii $R_{1}$ and $R_{2}$ of curvature these are $H=1 / R_{1}+1 / R_{2}$ and $K=1 / R_{1} R_{2}$. The parameter $\mu_{0}$ in (11) is the surface tension, $\kappa_{0}$ the bending rigidity, and $\bar{\kappa}_{0}$ its Gaussian counterpart. The geometric quantities appearing in the integral (11) are invariant under translations and rotations in space. They are also independent of the parametrization of the surface. We ignore a possible spontaneous curvature term linear in $H$. The energy of a physical membrane contains all higher powers in the principal radii of curvature, but these are irrelevant at large length scales. (In the language of renormalization group analysis, the first term is relevant, the second and third are marginal.)

The statistical behavior of fluctuating membranes was first studied by Helfrich [3] using only curvature terms. We allow for an additional surface term in (11) because flucutations arising from the curvature terms not only renormalize the bending rigidities, but also the tension. The thermal fluctuations soften the bending rigidity at large length scales, reducing it from the bare value $\kappa_{0}$ as follows

$$
\kappa_{\mathrm{eff}}=\kappa_{0}-\frac{\alpha}{4 \pi \beta} \ln (\Lambda L),
$$

where $\beta$ is the inverse temperature, $\Lambda$ is an ultraviolet momentum cutoff of the order of the inverse microscopic length scale $a$ given by the length of the molecules, whereas $L$ is an infrared cutoff determined by the finite size of the membrane. Various authors derived different values for $\alpha$, first $\alpha=1$ [ [4] was obtained, later $\alpha=3$ [7 10]. The second result has also been found in computer simulations [11]. For either value of $\alpha$, the rigidity disappears at length scales larger than the persistence length [12]

$$
\xi \sim a \exp \left(\frac{4 \pi}{\alpha} \beta \kappa_{0}\right)
$$

beyond which the normal vectors of the surface become uncorrelated-the surface looks crumpled. More recent calculations [13] suggest the value $\alpha=-1$, implying a stiffening instead of a softening of the bending rigidity. This new result was argued to arise from the use of another integration measure which respects the incompressible-fluid nature of the membrane from the outset. This is in contrast to previous studies of in-plane fluid [10] and elastic effects [14] which did not show any change in the value $\alpha=3$ (they only enter at the two-loop level [15]).

The renormalization of the Gaussian rigidity $\bar{\kappa}_{0}$ was first calculated in [9] to have the same form as in (2), but with $\alpha \rightarrow \bar{\alpha}=$ $-10 / 3$. This value is changed by in-plane fluid and elastic effects [10,14.

\footnotetext{
*E-mail: borelli@physik.fu-berlin.de

${ }^{\dagger}$ E-mail: kleinert@physik.fu-berlin.de; website: http://www.physik.fu-berlin/ kleiner

${ }^{\ddagger}$ E-mail: schakel@physik.fu-berlin.de
} 
The renormalization group flow of $\kappa_{\text {eff }}$ extracted from the one-loop result (2) has no nontrivial fixed point. If this conclusion persists to all orders in perturbation theory, it would imply the absence of a smooth phase with long-ranged correlations. The smooth appearance of lipid vesicles in the laboratory can then only be explained by their very large persistence length. An alternative explanation has recently been proposed in [16], where it was argued that the neglected higher order terms in the energy (1) may give rise to a nonperturbative mechanism, by which the crumpled phase can go over into a smooth phase via a sequence of two Kosterlitz-Thouless phase transitions or a single first-order one.

The renormalization of the surface tension has also been investigated by several authors. The results can be summarized by the formula

$$
\mu_{\mathrm{eff}}=\mu_{0}+\frac{\alpha^{\prime}}{4 \pi \beta} \frac{\mu_{0}}{\kappa_{0}} \ln (\Lambda L),
$$

with the value $\alpha^{\prime}=1$ found in [8,17] and $\alpha^{\prime}=3$ in [7],9]. In Ref. [18], an attempt was made to reconcile the differences. An almost planar surface without overhangs was considered in the Monge parametrization. The points on the surface are then specified by a vector field $\mathbf{r}(x)=\left(x_{1}, x_{2}, \phi(x)\right)$, where $\phi(x)$ denotes the vertical displacement of the surface with respect to a base plane with Cartesian coordinates $x=\left(x_{1}, x_{2}\right)$. For a surface with fixed topology, the Gaussian curvature energy is a constant and can be ignored. The remainder of Eq. (1) was expanded to fourth order in the displacement field. The relative weights of the resulting terms are fixed by their covariant origin. The authors encountered considerable problems in showing that this remains true after including the thermal fluctuations. They studied the renormalization of the surface tension by determining the coefficient $\tau$ of the first (constant) term in the expansion of the surface energy,

$$
\begin{aligned}
\tau & =\mu_{0}+\frac{1}{2 \beta} \int \frac{\mathrm{d}^{2} k}{(2 \pi)^{2}} \ln \left(\mu_{0} k^{2}+\kappa_{0} k^{4}\right) \\
& =\mu_{0}+\frac{1}{4 \pi \beta} \frac{\mu_{0}}{\kappa_{0}} \ln (\Lambda L)+c_{1} \Lambda^{2}+c_{2} \Lambda^{2} \ln \Lambda+c_{3}
\end{aligned}
$$

with $c_{i}$ constants, and comparing it with the coefficient $\mu_{\mathrm{eff}}$ of the second term [proportional to $(\partial \phi)^{2}$ ],

$$
\begin{aligned}
\mu_{\mathrm{eff}} & =\mu_{0}-\frac{1}{2 \beta} \int \frac{\mathrm{d}^{2} k}{(2 \pi)^{2}}\left[3-\frac{\mu_{0} k^{2}}{\mu_{0} k^{2}+\kappa_{0} k^{4}}\right] \\
& =\mu_{0}+\frac{1}{4 \pi \beta} \frac{\mu_{0}}{\kappa_{0}} \ln (\Lambda L)+c_{4} \Lambda^{2}+c_{5} .
\end{aligned}
$$

The above expressions differ from each other by positive powers of the cutoff $\Lambda$. Since the covariance of the theory implies $\tau=\mu_{\text {eff }}$, two factors were added to the energy, in order to correct Eq. (6). The first one corresponds to the Faddeev-Popov determinant associated with fixing the gauge. In the Monge gauge, its contribution is proportional to the cutoff $\Lambda$. The second (more ad hoc) factor introduces a nonlinear correction to the integration measure in the partition function. It accounts for the difference between an infinitesimal surface element on the membrane and its projection on the reference plane. This second correction factor, too, contributes only with positive powers of the cutoff. Added to the first one, it leads to the equality $\mu_{\mathrm{eff}}=\tau$, which is the main result of Ref. [18].

Motivated by these problems, and by the renewed interest in the subject, we study the role of thermal fluctuations in a more general approach. Employing a derivative expansion [19], we calculate the full effective energy functional produced by Gaussian fluctuations for an arbitrary background configuration, maintaining the full nonlinear structure of the energy at all intermediate steps.

The mean curvature in the Monge parametrization reads

$$
H=\partial \cdot N=\partial_{\mu} N_{\mu}
$$

where the summation is over the first two components only $(\mu=1,2) ; \mathbf{N}$ is the unit normal to the surface

$$
\mathbf{N}=\frac{1}{\sqrt{1+(\partial \phi)^{2}}}\left(-\partial_{1} \phi,-\partial_{2} \phi, 1\right)
$$

and the surface elements are

$$
\mathrm{d} S=\mathrm{d}^{2} x \sqrt{1+(\partial \phi)^{2}}
$$

so that the first two terms of the energy (1) read explicitly 


$$
E_{0}[\phi]=\int \mathrm{d}^{2} x \sqrt{1+(\partial \phi)^{2}}\left\{\mu_{0}+\frac{\kappa_{0}}{2}\left[\frac{\left(\partial^{2} \phi\right)^{2}}{1+(\partial \phi)^{2}}-2 \frac{\partial_{\mu} \phi \partial_{\nu} \phi \partial_{\mu} \partial_{\nu} \phi \partial^{2} \phi}{\left[1+(\partial \phi)^{2}\right]^{2}}+\frac{\left(\partial_{\mu} \phi \partial_{\nu} \phi \partial_{\mu} \partial_{\nu} \phi\right)^{2}}{\left[1+(\partial \phi)^{2}\right]^{3}}\right]\right\}
$$

Physically, $\mu_{0}$ corresponds to the chemical potential specifying the exchange of molecules between the (incompressible) membrane and its aqueous environment.

The main purpose of this note is to show that the ultraviolet divergent parts of the one-loop corrections induced by thermal fluctuations are of precisely the same form as in (10), and in particular, that the three terms in the curvature energy renormalize in the same way, resulting in an overall renormalization of $\kappa_{0}$ alone.

To apply the derivative expansion we write the partition function as a functional integral over the displacement field

$$
Z=\int \mathrm{D} \phi \exp \left(-\beta E_{0}\right)
$$

with each field configuration weighted with a Boltzmann factor. Fixing a gauge is generally accompanied by a Faddeev-Popov determinant appearing in the measure of the functional integral. Following Ref. [20] we adopt dimensional regularization to handle momentum integrals which diverge in the ultraviolet. This is common practice in the technically closely related nonlinear sigma model. The great advantage of dimensional regularization over regularization with a momentum cutoff $\Lambda \sim 1 / a$, is that terms diverging with a strictly positive power of $\Lambda$ are suppressed. As a result, both the Faddeev-Popov determinant corresponding to the Monge gauge and the second correction factor introduced in Ref. [18], which contain only positive powers of the cutoff, are unity in dimensional regularization, and the difficulties addressed in that reference are avoided. Only logarithmic divergences show up as poles in $\epsilon$, where $\epsilon=2-D, D$ being the dimension of the membrane. The connection between the two types of regularization is

$$
\frac{1}{\epsilon} \rightarrow \ln (\Lambda L)
$$

with the linear size $L$ of the membrane representing the relevant long-distance scale. The rationale for using dimensional regularization is that contributions to the effective energy with strictly positive powers of the ultraviolet cutoff are connected to $\delta^{(2)}(x=0)$. These highly local terms are uninteresting at large lenght scales [21,22].

In the one-loop approximation, the exponent in (11) may be expanded up to second order around a background configuration $\Phi(x)$ extremizing $E_{0}$. A nontrivial background requires the presence of an extra source term. For brevity, this term will not be written down explicitly when setting $\delta E_{0} / \delta \Phi=0$. The resulting integral is Gaussian and yields an effective energy

$$
E_{\text {eff }}[\Phi]=E_{0}[\Phi]+E_{1}[\Phi]=E_{0}[\Phi]+\frac{1}{2 \beta} \operatorname{Tr} \ln \left[\left.\frac{\delta^{2}\left(\beta E_{0}\right)}{\delta \phi(x) \delta \phi(y)}\right|_{\Phi}\right]
$$

where the expression in square brackets corresponds to the matrix of second functional derivatives of $E_{0}$ and the trace $\operatorname{Tr}$ stands for the trace of this matrix, i.e., the integral $\int \mathrm{d}^{2} x$ over space, as well as the integral $\int \mathrm{d}^{2} k /(2 \pi)^{2}$ over momentum [19].

The one-loop correction $E_{1}[\Phi]$ to the energy will now be calculated in a derivative expansion for a nearly flat, but arbitrarily tilted background configuration. The expansion has the general form

$$
E_{1}[\Phi]=\int \mathrm{d}^{2} x\left[\mathcal{V}\left(V_{\lambda}\right)+\mathcal{Z}^{1}\left(V_{\lambda}\right)\left(\partial_{\mu} V_{\mu}\right)^{2}+\mathcal{Z}_{\mu \nu}^{2}\left(V_{\lambda}\right) \partial_{\mu} V_{\nu} \partial_{\sigma} V_{\sigma}+\mathcal{Z}_{\mu \nu \sigma \rho}^{3}\left(V_{\lambda}\right) \partial_{\mu} V_{\nu} \partial_{\sigma} V_{\rho}+\cdots\right]
$$

where we introduced the abbreviation $V_{\mu}=\partial_{\mu} \Phi$, while $\mathcal{V}, \mathcal{Z}^{1}, \mathcal{Z}_{\mu \nu}^{2}$, and $\mathcal{Z}_{\mu \nu \sigma \rho}^{3}$ are functions of $V_{\mu}$ to be determined. Following Ref. [19], we set $V_{\mu}(x)=\bar{V}_{\mu}+v_{\mu}(x)$, where $\bar{V}_{\mu}$ denotes the constant part of $V_{\mu}(x)$, and expand Eq. (14) in powers of $v_{\mu}(x)$ and its derivatives, to obtain

$$
\begin{aligned}
E_{1}\left[\bar{V}_{\lambda}+v_{\lambda}\right]=\int \mathrm{d}^{2} x[ & \mathcal{V}\left(\bar{V}_{\lambda}\right)+\frac{\partial \mathcal{V}\left(\bar{V}_{\lambda}\right)}{\partial \bar{V}_{\mu}} v_{\mu}+\frac{1}{2} \frac{\partial^{2} \mathcal{V}\left(\bar{V}_{\lambda}\right)}{\partial \bar{V}_{\mu} \partial \bar{V}_{\nu}} v_{\mu} v_{\nu}+\mathcal{Z}^{1}\left(\bar{V}_{\lambda}\right)\left(\partial_{\mu} v_{\mu}\right)^{2} \\
& \left.+\mathcal{Z}_{\mu \nu}^{2}\left(\bar{V}_{\lambda}\right) \partial_{\mu} v_{\nu} \partial_{\sigma} v_{\sigma}+\mathcal{Z}_{\mu \nu \sigma \rho}^{3}\left(\bar{V}_{\lambda}\right) \partial_{\mu} v_{\nu} \partial_{\sigma} v_{\rho}+\cdots\right]
\end{aligned}
$$

with space-independent $\mathcal{V}\left(\bar{V}_{\lambda}\right)$ and $\mathcal{Z}\left(\bar{V}_{\lambda}\right)$ 's. These functions will now be extracted from the expansion of the $\operatorname{Tr} \ln$ in $(13)$ up to quadratic terms in $v_{\mu}$ and $\partial_{\mu} v_{\nu}$.

The functional derivatives in (13) are calculated using the Euler-Lagrange formula

$$
\frac{\delta F[\phi]}{\delta \phi(x)}=\frac{\partial f}{\partial \phi}-\partial_{\mu} \frac{\partial f}{\partial\left(\partial_{\mu} \phi\right)}+\partial_{\mu} \partial_{\nu} \frac{\partial f}{\partial\left(\partial_{\mu} \partial_{\nu} \phi\right)}+\cdots
$$


with $F[\phi]=\int \mathrm{d}^{2} x f\left(\phi, \partial_{\mu} \phi, \partial_{\mu} \partial_{\nu} \phi, \ldots\right)$. To keep track of the many terms appearing in the resulting expression we have used the algebraic computer program FORM [23].

We consider first the renormalization of the surface tension. Since the energy density $\sqrt{1+V^{2}}$ does not contain derivatives of $V_{\mu}$, we may set $v_{\mu}(x)$ to zero and consider $E_{1}\left[\bar{V}_{\lambda}\right]$ only,

$$
\beta E_{1}\left[\bar{V}_{\lambda}\right]=-\frac{1}{4} \operatorname{Tr} \ln \left(1+\bar{V}^{2}\right)+\frac{1}{2} \operatorname{Tr} \ln \left[G^{-1}(p)\right]
$$

Here, $G^{-1}(p)$ denotes the inverse propagator:

$$
G^{-1}(p)=\left(\mu_{0}+\kappa_{0} p^{2}\right) p^{2}-\left(\mu_{0}+2 \kappa_{0} p^{2}\right)(\bar{U} \cdot p)^{2}+\kappa_{0}(\bar{U} \cdot p)^{4}
$$

where $\bar{U}_{\mu}$ is the constant vector

$$
\bar{U}_{\mu}=\frac{\bar{V}_{\mu}}{\sqrt{1+V^{2}}}
$$

In dimensional regularization, the first term at the right-hand side of (17) is zero. To evaluate the remaining Tr ln, we apply a standard trick and first differentiate $(17)$ with respect to $\mu_{0}$ to obtain

$$
\frac{\partial E_{1}\left[\bar{V}_{\lambda}\right]}{\partial \mu_{0}}=\frac{1}{2 \beta} \operatorname{Tr}\left[\frac{p^{2}-(\bar{U} \cdot p)^{2}}{\left(\mu_{0}+\kappa_{0} p^{2}\right) p^{2}-\left(\mu_{0}+2 \kappa_{0} p^{2}\right)(\bar{U} \cdot p)^{2}+\kappa_{0}(\bar{U} \cdot p)^{4}}\right]
$$

where $p_{\mu}=-i \partial_{\mu}$. Because the integrand contains no space-dependence, the spatial part of the trace in (20) yields an area factor $A=\int \mathrm{d}^{D} x$, and we are left with the momentum integral

$$
\frac{\partial E_{1}\left[\bar{V}_{\lambda}\right]}{\partial \mu_{0}}=\frac{A}{2 \beta} \int \frac{\mathrm{d}^{D} k}{(2 \pi)^{D}}\left[\frac{k^{2}-(\bar{U} \cdot k)^{2}}{\left(\mu_{0}+\kappa_{0} k^{2}\right) k^{2}-\left(\mu_{0}+2 \kappa_{0} k^{2}\right)(\bar{U} \cdot k)^{2}+\kappa_{0}(\bar{U} \cdot k)^{4}}\right] .
$$

Being interested only in the ultraviolet divergent terms, we obtain, in dimensional regularization

$$
\frac{\partial E_{1}\left[\bar{V}_{\lambda}\right]}{\partial \mu_{0}}=\frac{1}{4 \pi \kappa_{0} \beta} \frac{1}{\epsilon} \int \mathrm{d}^{2} x \sqrt{1+\bar{V}^{2}}
$$

After integrating again with respect to $\mu_{0}$ and comparing the result with (14) we find (up to an irrelevant additive constant)

$$
\mathcal{V}\left(V_{\lambda}\right)=\frac{\mu_{0}}{4 \pi \beta \kappa_{0}} \frac{1}{\epsilon} \sqrt{1+V^{2}}=\frac{\mu_{0}}{4 \pi \beta \kappa_{0}} \frac{1}{\epsilon} \sqrt{1+(\partial \Phi)^{2}}
$$

where we replaced $\bar{V}_{\lambda}$ with the full background field $V_{\lambda}(x)$, to obtain the first term in (14). Note that this one-loop correction is precisely of the same form as the surface term contained in the original energy expression (1). This term can consequently be combined with the original one by introducing the renormalized tension

$$
\mu_{\mathrm{eff}}=\mu_{0}+\frac{1}{4 \pi \beta} \frac{\mu_{0}}{\kappa_{0}} \frac{1}{\epsilon} .
$$

This result, corresponding to $\alpha^{\prime}=1$ in (4), is in agreement with Refs. [8, 17], but disagrees with Refs. [7, 9] where the value $\alpha^{\prime}=3$ was obtained. To understand the differences, we note that in these last two references, the energy (1) with $\mu_{0}=0$ was used instead. That is, the renormalization of the surface tension calculated by these authors was generated solely by the curvature terms. However, the surface term also contributes. In fact, it generates a contribution with $\alpha^{\prime}=-2$, which, together with the contribution obtained from the curvature terms, results in the value $\alpha^{\prime}=1$. This is also the value obtained in Ref. [18], as can be seen by disregarding the terms proportional to positive powers of $\Lambda$ in Eq. (5) and using the relation (12). There, the covariance of the first two terms in the expansion of the surface energy is assured by introducing correction factors proportional to positive powers of the cutoff. Our result, based on dimensional regularization where terms with positive powers of the cutoff are suppressed, proves the covariance of all terms in the expansion of the surface energy since the full expression has been maintained.

We continue to investigate the renormalization of the bending rigidity. Since the three terms involved contain derivatives of the background field $V_{\mu}$, we now have to employ the derivative expansion. As a first step, we Taylor expand the logarithm in (13) as:

$$
\beta E_{1}\left[\bar{V}_{\lambda}+v_{\lambda}(x)\right]-\beta E_{1}\left[\bar{V}_{\lambda}\right]=\frac{1}{2} \operatorname{Tr} \ln [1+G(p) \Lambda(x, p)]=\frac{1}{2} \operatorname{Tr}[G(p) \Lambda(p, x)]-\frac{1}{4} \operatorname{Tr}[G(p) \Lambda(x, p) G(p) \Lambda(x, p)]+\cdots,
$$


where $G(p)$ is the propagator defined in $(18)$ and $\Lambda(x, p)$ contains the $x$-dependent terms obtained from functionally differentiating $E_{0}$ twice, setting $\partial_{\mu} \Phi(x)=V_{\mu}(x)=\bar{V}_{\mu}+v_{\mu}(x)$ and expanding up to second order in $v_{\mu}$ and $\partial_{\mu} v_{\nu}$.

The first term in (25) can be calculated in a similar fashion as $\mathcal{V}\left(V_{\lambda}\right)$. In the second term, all momentum operators have to be moved to the left [19], by repeatedly applying the identity

$$
f(x) p_{\mu} g(x)=\left(p_{\mu}+i \partial_{\mu}\right) f(x) g(x),
$$

where $f(x)$ and $g(x)$ are arbitrary functions and the derivative $\partial_{\mu}$ acts only on the next object to the right, while the derivative $p_{\mu}$ acts on everything to the right.

The typical momentum integrals showing up at the one-loop order are of the form

$$
I_{m, n}=\int \frac{\mathrm{d}^{D} k}{(2 \pi)^{D}} k^{m} G^{n}(k) \sim \int \mathrm{d} k\left\{\begin{array}{ll}
k^{m+D-1-2 n} & \text { infrared } \\
k^{m+D-1-4 n} & \text { ultraviolet }
\end{array},\right.
$$

with $m, n>0$. They diverge in the infrared when $m+D-1-2 n \leq-1$, and in the ultraviolet when $m+D-1-4 n \geq-1$. For $D=2$ these conditions become $m-2 n \leq-2, m-4 n \geq-2$, respectively, and the two types of divergences are seen to be separated by a wedge of finite integrals in the $(m, n)$-plane starting at $(-2,0)$.

After a tedious and lengthy calculation, involving of the order of $10^{4}$ terms, done with help of a program written in FORM [23], we obtained the divergent terms to second order in derivatives of the field $v_{\mu}$ :

$$
\begin{aligned}
\beta E_{1}\left[\bar{V}_{\lambda}+v_{\lambda}\right]-\beta E_{1}\left[\bar{V}_{\lambda}\right]=\int \mathrm{d}^{2} x\{ & \frac{\mu_{0}}{4 \pi \kappa_{0}} \frac{1}{\epsilon}\left[\frac{\bar{V}_{\mu}}{\left(1+\bar{V}^{2}\right)^{1 / 2}} v_{\mu}+\frac{1}{2}\left(\frac{\delta_{\mu \nu}}{\left(1+\bar{V}^{2}\right)^{1 / 2}}+\frac{\bar{V}_{\mu} \bar{V}_{\nu}}{\left(1+\bar{V}^{2}\right)^{3 / 2}}\right) v_{\mu} v_{\nu}\right] \\
& -\frac{3}{8 \pi} \frac{1}{\epsilon}\left[\frac{1}{\left(1+\bar{V}^{2}\right)^{1 / 2}}\left(\partial_{\mu} v_{\mu}\right)^{2}-2 \frac{\bar{V}_{\mu} \bar{V}_{\nu}}{\left(1+\bar{V}^{2}\right)^{3 / 2}} \partial_{\mu} v_{\nu} \partial_{\sigma} v_{\sigma}+\frac{\bar{V}_{\mu} \bar{V}_{\nu} \bar{V}_{\sigma} \bar{V}_{\rho}}{\left(1+\bar{V}^{2}\right)^{5 / 2}} \partial_{\mu} v_{\nu} \partial_{\sigma} v_{\rho}\right] \\
& \left.-\frac{1}{4 \pi} \frac{1}{\epsilon_{\mathrm{ir}}}\left[\frac{\bar{V}_{\mu} \bar{V}_{\nu}}{\left(1+\bar{V}^{2}\right)^{3 / 2}} \partial_{\mu} v_{\nu} \partial_{\sigma} v_{\sigma}-\frac{\bar{V}_{\mu} \bar{V}_{\nu} \bar{V}_{\rho} \bar{V}_{\sigma}}{\left(1+\bar{V}^{2}\right)^{5 / 2}} \partial_{\mu} v_{\nu} \partial_{\sigma} v_{\rho}\right]\right\}
\end{aligned}
$$

In deriving this expression we also encountered infrared divergences. These are regularized in the same scheme as used to regularize the ultraviolet divergences. To distinguish the two we gave epsilon an index ir in case of an infrared divergence. We leave the discussion of the infrared divergences to the next paragraph, and first analyze the ultraviolet ones. Comparing (28) to (15) with $\mathcal{V}\left(\bar{V}_{\lambda}\right)$ given by (23), we see that the terms proportional to $\mu_{0}$ precisely correspond to the first two terms at the right-hand side of (15), as it should be. Moreover, we conclude that the $\mathcal{Z}$-functions in (15) are given by

$$
\mathcal{Z}^{1}\left(\bar{V}_{\lambda}\right)=-\frac{3}{8 \pi \beta} \frac{1}{\epsilon} \frac{1}{\left(1+\bar{V}^{2}\right)^{1 / 2}}, \mathcal{Z}_{\mu \nu}^{2}\left(\bar{V}_{\lambda}\right)=\frac{3}{4 \pi \beta} \frac{1}{\epsilon} \frac{\bar{V}_{\mu} \bar{V}_{\nu}}{\left(1+\bar{V}^{2}\right)^{3 / 2}}, \mathcal{Z}_{\mu \nu \sigma \rho}^{3}\left(\bar{V}_{\lambda}\right)=-\frac{3}{8 \pi \beta} \frac{1}{\epsilon} \frac{\bar{V}_{\mu} \bar{V}_{\nu} \bar{V}_{\sigma} \bar{V}_{\rho}}{\left(1+\bar{V}^{2}\right)^{5 / 2}} .
$$

By replacing the constant $\bar{V}_{\lambda}$ with the full background field $V_{\mu}(x)=\partial_{\mu} \Phi(x)$, we obtain for the divergent parts of the expansion (14) the explicit form

$$
\beta E_{1}[\Phi]=\frac{1}{4 \pi \epsilon \kappa_{0}} \int \mathrm{d}^{2} x \sqrt{1+(\partial \Phi)^{2}}\left\{\mu_{0}-\frac{3 \kappa_{0}}{2}\left[\frac{\left(\partial^{2} \Phi\right)^{2}}{1+(\partial \Phi)^{2}}-2 \frac{\partial_{\mu} \Phi \partial_{\nu} \Phi \partial_{\mu} \partial_{\nu} \Phi \partial^{2} \Phi}{\left[1+(\partial \Phi)^{2}\right]^{2}}+\frac{\left(\partial_{\mu} \Phi \partial_{\nu} \Phi \partial_{\mu} \partial_{\nu} \Phi\right)^{2}}{\left[1+(\partial \Phi)^{2}\right]^{3}}\right]\right\} .
$$

We see that the thermally generated terms at the one-loop level are precisely of the same form as those present in the original energy expression (10). In addition, the relative weights of the curvature terms produced by the fluctuations are the same as those found there. They can therefore be combined with the original terms by introducing the renormalized rigidity

$$
\kappa_{\mathrm{eff}}=\kappa_{0}-\frac{3}{4 \pi \beta} \frac{1}{\epsilon}
$$

whose value is in agreement with [7 11].

As seen in (28), the one-loop corrections seem to have introduced infrared divergences in the theory. A closer inspection reveals that the infrared-divergent contributions all stem from the surface energy term in (10), so that it suffices to analyze the one-loop corrections to the truncated energy

$$
E_{0}^{\prime}=\mu_{0} \int \mathrm{d}^{2} x \sqrt{1+(\partial \phi)^{2}} .
$$


Infrared divergences in this model have previously been studied in [24], where they were shown to disappear for an infinitely small dimension $D$ of the membrane to all orders in $D$. In our calculation the problem arises for $D=2-\epsilon$. When calculating the effective action, we expand (32) around the background field $\Phi$ extremizing $E_{0}^{\prime}$, i.e.,

$$
\left.\frac{\delta E_{0}^{\prime}}{\delta \phi}\right|_{\Phi}=0
$$

which reads explicitly

$$
\frac{\partial^{2} \Phi}{\left[1+(\partial \Phi)^{2}\right]^{1 / 2}}-\frac{\partial_{\rho} \Phi \partial_{\sigma} \Phi \partial_{\sigma} \partial_{\rho} \Phi}{\left[1+(\partial \Phi)^{2}\right]^{3 / 2}}=0 .
$$

The presence of the implicitly assumed sources turns this equation in a nontrivial one. Rewriting $\partial_{\mu} \Phi(x)=\bar{V}_{\mu}+v_{\mu}(x)$, expanding to linear order in $v_{\mu}$, and substituting the resulting expression in (28), we see the infrared divergences to vanish for a two-dimensional membrane.

In conclusion, we have demonstrated that all logarithmically divergent one-loop corrections induced by thermal fluctuations are precisely of the same form as in the original energy $(10)$, so that they can be removed by a renormalization of the surface tension and bending rigidity.

\section{ACKNOWLEDGMENTS}

We thank V. Schulte-Frohlinde and S. Shabanov for useful discussions, and W. Helfrich and H. A. Pinnow for sharing their results prior to publication.

[1] Statistical Mechanics of Membranes and Surfaces, Proceedings of the Fifth Jerusalem Winter School for Theoretical Physics, 1987/1988, edited by D. Nelson, T. Piran, and S. Weinberg (World Scientific, Singapore, 1989).

[2] P. B. Canham, J. Theor. Biol. 26, 61 (1970).

[3] W. Helfrich, Z. Naturforsch. 28c, 693 (1973).

[4] W. Helfrich, J. Phys. (Paris) 46, 1263 (1985).

[5] W. Helfrich, J. Phys. (Paris) 48, 285 (1987).

[6] D. Förster, Europhys. Lett. 4, 65 (1987).

[7] L. Peliti and S. Leibler, Phys. Rev. Lett. 54, 1690 (1985).

[8] D. Förster, Phys. Lett. A 114, 115 (1986).

[9] H. Kleinert, Phys. Lett. A 114, 263 (1986).

[10] H. Kleinert, J. Stat. Phys. 56, 227 (1989).

[11] G. Gompper and D. M. Kroll, J. Phys. I France 6, 1305 (1996).

[12] P. G. de Gennes and C. Taupin, J. Phys. Chem. 86, 2294 (1982).

[13] W. Helfrich, Eur. Phys. J B 1, 481 (1998); H. A. Pinnow and W. Helfrich, Effect of Thermal Undulations on the Bending Elasticity and Spontaneous Curvature of Fluid Membranes, Berlin preprint (1998).

[14] S. Ami and H. Kleinert, Phys. Lett. A 120, 207 (1987).

[15] F. David in Ref. [1].

[16] H. Kleinert, Smooth Membranes and their Spiky Phases, Berlin preprint (1998).

[17] F. David and S. Leibler, J. Phys. II France 1, 959 (1991).

[18] W. Cai, T. C. Lubensky, P. Nelson, and T. Powers, J. Phys. II France 4, 931 (1994).

[19] C. M. Fraser, Z. Phys. C 28, 101 (1985).

[20] S. Leibler, in [1].

[21] T. Y. Cao and S. S. Schweber, Synthese 97 (1993); in Renormalization, edited by L. M. Brown (Springer-Verlag, Berlin, 1993).

[22] J. F. Donoghue, Phys. Rev. D 50, 3874 (1994); e-print gr-qc/9512024 (1995).

[23] J. A. M. Vermaseren, FORM Version 1, software freely available from ftp.nikhef.nl in /pub/form.

[24] F. David, Phys. Lett. B 102, 193 (1981). 